\title{
Snowballs in Euclid and WFIRST Detectors
}

\author{
Analia N. Cillis*a , Dave A. Cottingham ${ }^{\mathrm{b}}$, Augustyn Waczynski ${ }^{\mathrm{c}}$, Robert J. Hill , Yiting Wen ${ }^{\mathrm{c}}$, \\ Roger Foltz' ${ }^{\mathrm{c}}$, Edward Cheng ${ }^{\mathrm{b}}$, Stephanie A. Cheung ${ }^{\mathrm{d}}$, Benjamin Cho ${ }^{\mathrm{d}}$, Gregory S. Delo ${ }^{\mathrm{d}}$, Ali R. \\ Feizi $^{\mathrm{f}}$, Michael Hickey ${ }^{\mathrm{c}}$, Tsuwei Hwang ${ }^{\mathrm{d}}$, Emily Kan ${ }^{\mathrm{c}}$, Eric Kan ${ }^{\mathrm{c}}$, Wayne B. Landsman ${ }^{\mathrm{g}}$, Jonathan \\ Mah $^{\mathrm{b}}$, Lane Meier ${ }^{\mathrm{b}}$, Chris Merchant ${ }^{\mathrm{e}}$, Laddawan Miko ${ }^{\mathrm{c}}$, Jhabvala D. Murzy ${ }^{\mathrm{c}}$, Dino Rossetti ${ }^{\mathrm{b}}$, Kyle A. \\ Turck $^{\mathrm{e}}$ \\ ${ }^{a}$ University of Maryland at Baltimore County, NASA Goddard Space Flight Center, Greenbelt \\ Road, Greenbelt, MD, USA 20071; ${ }^{\mathrm{b}}$ Conceptual Analytics; ${ }^{\mathrm{c}}$ NASA Goddard Space Flight Center; \\ ${ }^{\mathrm{d}} \mathrm{AS}$ and D, Inc; ${ }^{\mathrm{e}} \mathrm{Global}$ Science \& Technology Inc; ${ }^{\mathrm{f}} \mathrm{AK}$ Aerospace Technology Corporation; \\ ${ }^{g}$ ADNET Systems Inc
}

\begin{abstract}
Snowballs are transient events observed in HgCdTe detectors with a sudden increase of charge in a few pixels. They appear between consecutive reads of the detector, after which the affected pixels return to their normal behavior. The origin of the snowballs is unknown, but it was speculated that they could be the result of alpha decay of naturally radioactive contaminants in the detectors, but a cosmic ray origin cannot be ruled out. Even though previous studies predicted a low rate of occurrence of these events, and consequently, a minimal impact on science, it is interesting to investigate the cause or causes that may generate snowballs and their impact in detectors designed for future missions. We searched for the presence of snowballs in the dark current data in Euclid and Wide Field Infrared Survey Telescope (WFIRST) detectors tested in the Detector Characterization Laboratory at Goddard Space Flight Center. Our investigation shows that for Euclid and WFIRST detectors, there are snowballs that appear only one time, and others that repeat in the same spatial localization. For Euclid detectors, there is a correlation between the snowballs that repeat and bad pixels in the operational masks (pixels that do not fulfill the requirements to pass spectroscopy noise, photometry noise, quantum efficiency, and/or linearity). The rate of occurrence for a snowball event is about 0.9 snowballs/hr. in Euclid detectors (for the ones that do not have associated bad pixels in the mask), and about 0.7 snowballs/hr. in PV3 Full Array Lot WFIRST detectors.
\end{abstract}

Keywords: Euclid, WFIRST, snowballs, MCT

\section{INTRODUCTION}

Several authors have noticed the appearance of snowball events in $\mathrm{HgCdTe}$ photodiode arrays in the past. These features were observed, for example, in Hubble Space Telescope's Wide Field Camera 3 (WFC3) detectors, and in the NIRSpec and NIRCam instruments of the James Webb Space Telescope (JWST). In all cases, they had low rates of occurrence, and it was very difficult to present a clear picture of what may be the source of the snowball. During the past years, several hypotheses have been proposed about its origin, from decay of radioactive contaminants to cosmic rays.

*analia.n.cillis@nasa.gov; phone 1301 286-5865; fax 1301 286-1672 
In our work, we investigated the presence of these artifacts in Euclid and WFIRST detectors. These detectors have been characterized during the last 3 years at NASA's Goddard Space Flight Center. Euclid is a European Space Agency (ESA) mission designed to accurately measure the expansion history of the universe and the growth of cosmic structures. The Euclid instruments are provided by the ESA member states, with NASA contributing to the NISP (near-infrared spectrometer and imaging photometer). WFIRST is a NASA observatory designed to perform wide-field imaging and spectroscopic surveys of the near infrared sky. The main goal of the mission is to answer essential questions in exoplanet and dark energy research and will advance topics ranging from galaxy evolution to the study of objects within the Galaxy and within the Solar System.

B. Hilbert ${ }^{1}$ searched for snowballs within two epochs of WFC3 data. The first data set was taken in the Detector Characterization Laboratory (55 hours) and the second came from the instrument thermal test (45 hours). He found a snowball rate between 0.4 and 0.8 snowballs per hour. A snowball affected between 11 and 34 pixels in those detectors, and contains between 200,000 and 900,000 electrons.

After compiling different pieces of evidence, P. McCullough ${ }^{2}$ extensively discussed the possibility that the snowballs could be originated by alpha particles emitted from either naturally radioactive thorium and/or uranium, and/or unknown radionuclides inside the $\mathrm{HgCdTe}$ detector, at $\sim 1 \mathrm{ppm}$ concentrations within the device.

Later, M. J. Durbin et al. ${ }^{3}$ created a catalog of 7392 snowball detections based on all in-flight WFC3 data ( 6200 hours of exposure time). They concluded that the rate at which snowballs were observed was approximately constant over time, suggesting that if the source of snowballs is radioactivity in the detector or bonding material, the primary contaminant is $\mathrm{U}-238$.

On the other hand, B. J. Rauscher, et al. ${ }^{4}$ observed that the snowball rate in the NIRSpec instrument of the JWST, went down with time, with a decay rate that is consistent with known radioactive isotopes that are used during the fabrication process at the detectors. They predicted that when JWST flies in 2018, the snowball rate would be negligible. (The JWST launch date has subsequently slipped and is now 2020).

B. Hilbert ${ }^{5}$ searched for snowballs in NIRCam ground testing data. Examining a total of at least 32.6 hours of exposure time for each of nine of the NIRCam detectors, he found that snowballs appear in the data at a rate of $0.2-0.6$ snowballs per hour. Typical snowballs in the shortwave detectors covered an area of roughly 250-400 pixels and contained 280,000 - 490,000 DN. On average, snowballs in the longwave detectors were roughly the same size, but brighter by about a factor of two. Several of the snowballs he observed were repeaters, falling on the same location of a previous snowball. He concluded that radioactive decay from within the detector material, may be the source of the snowballs.

For WFIRST engineering detectors 18241,18242 , and 18244, D. Cottingham ${ }^{6}$ reported a rate between 0.5 and 1 snowballs per hr., for snowballs with charge deposited larger than $10^{5}$ electrons and at least 9 pixels affected. The method of analysis is similar to this paper. In addition, data from engineering MCT detectors manufactured by Raytheon, and from a total of 208 hours of darks on four different detectors found a rate of 0.03 to 0.2 snowballs $/ \mathrm{hr}$ for deposited charge greater than $10^{5}$ electrons.

In this work, we quantified the presence of snowballs in Euclid and WFIRST detectors. We discussed the rate, pixels affected, total charge deposited, spatial distribution, and if the snowballs repeat or not at the same localization. With the goal to find out the origin of snowballs events, we analyzed their correlation with bad pixels in the operational mask (for Euclid detectors), and we compared snowballs with cosmic ray events, to evaluate if the first could be subsets of the second.

This paper is organized as follows: in the next section, we describe the data used for our snowball search; in section 3 , we outline the method and algorithm used in our search; section 4 compiles our findings for Euclid and WFIRST detectors. Finally, we discuss our results in section 5. 


\section{DATA COLLECTION}

The data used for this analysis was collected in the Detector Characterization Laboratory (DCL) of NASA's Goddard Space Flight Center (GSFC) between 2015 and 2018.

In the case of Euclid detectors, we searched for snowballs in the data from Sensor Chip Subassemblies (SCS) classified as "flight grade". Each SCS consists of a Sensor Chip Assembly (SCA), SCE/SIDECAR ASIC, connected together with a Cryo Flex Cable (CFC). Euclid detectors are 2048x2048 HgCdTe photodiode arrays with pixel pitch of $18 \mu \mathrm{m}$. The detectors are surrounded by a band of reference pixels. These pixels are not light sensitive and are used to correct the remaining 2040x2040 pixels. All Euclid flight detectors were tested in the Big Dewar of the DCL, which allows simultaneous testing of four SCS. We performed six Flight Runs (FR), with each run consisting of four SCS. The Euclid Flight detectors were operated at a temperature of $100 \mathrm{~K}$, a detector bias of $0.5 \mathrm{~V}$, a gain setting of $15 \mathrm{~dB}$, and a frame time of 1.4548 seconds. In Flight Run 2, one of the detectors, SCS 18275_005-2_0007F020, was an engineering unit. After the initial cool down and optimization of all SCS units it was determined that the engineering SCS did not function at the operating temperature, and it was powered down to enable testing of the remaining detectors. In Flight Run 6, one of the units incorporated the newly designed and assembled Option B SCE, but with a non-flight SCA. We did not perform snowball searches in either of these two detectors.

We searched for snowballs in 2-hour dark current data, to assure low background signal. Each exposure of dark data consisted of 10 saved frames with 549 dropped frames between saved frames, uniformly spaced over 2 hours. Table 1 summarizes the total number of hours where we searched for snowballs in each Flight Run of Euclid detectors.

The WFIRST detectors are 4096x4096 HgCdTe photodiode arrays with pixel pitch of $10 \mu \mathrm{m}$. As with the Euclid detectors, the image pixels in WFIRST detectors (4088x4088 array) are surrounded by a band of reference pixels. We performed several tests of WFIRST detectors in different Dewars at the DCL (HyC, IR4, and JMAPS), at different temperatures and biases. For our snowball search, we only used the 2-hour dark current data collected at $100 \mathrm{~K}$ and detector bias of $1.0 \mathrm{~V}$, with a frame time of 5.4 seconds. For each 2-hour dark exposure, we took 101 frames with 13 drops between frames uniformly spaced over 2 hours of exposure time (sample up the ramp).

We looked for snowballs in the WFIRST PV3 full array lot detectors. This lot consisted of 13 engineering-grade SCAs, but only 11 were analyzed for snowballs due to performance problems with two detectors. Table 6 shows the total number of hours of dark current data used to search for snowballs in each WFIRST detector.

We also looked for snowballs in the engineering WFIRST PV2A detector 18138 that was tested several times between August 2016 and May 2017 at the DCL. We analyzed a total of 94 hours of dark current for this detector.

\section{METHOD AND ALGORITHM APPLIED}

For each ramp of 2-hour dark current data, for both Euclid and WFIRST detectors, we applied the standard method of reference pixel correction and conversion gain.

We selected a snowball event if it met the following criteria: the value of the pixel was above the Gaussian mean plus 50 times the Gaussian standard deviation of the background signal level in the frame; the pixel exceeded this threshold less than 70 percent of the time (to avoid hot pixels); the event was kept only if the 4 nearest neighbor pixels were above the Gaussian mean plus 25 times the Gaussian standard deviation of the signal level in the frame; and if the second nearest 
neighbors were above the Gaussian mean plus 10 times the Gaussian standard deviation in the frame. We only reported results if the total charge collected in the event was greater than $10^{5}$ electrons, and the size of the snowball was larger than 9 pixels. To count an event as a snowball we considered only those that are circular in shape, and not the ones that appear elongated, as in cosmic rays impacts.

For all cases, after applying this algorithm to the data, we performed a visual check to verify the presence of a snowball event.

We computed for each snowball its localization, number of pixels affected, total charge, and the time when the snowball appeared. We also recorded if the snowball repeated at the same spatial localization. For the case of the events that repeated in the same position, we looked at the flat field to see if these events corresponded to any visible feature in the detector.

In the case of Euclid detectors, we also took into account if the central pixels in the snowballs were correlated to bad pixels in the operational mask (pixels that do not fulfill the requirements to pass spectroscopy noise, photometry noise, quantum efficiency, and/or linearity).

For the Euclid detectors of Flight Run 3, we also searched for cosmic rays traces and compared them with the snowballs found in the same detectors. As with snowballs, we performed a visual check for each of the cosmic rays found. The algorithm to search for cosmic rays was similar to the one used for our snowball compilation, but instead of looking for events of circular shapes we searched for elongated events where one axis was larger than twice the other axis, and as for snowballs, we recorded the cosmic ray events with deposited charge larger than $10^{5}$ electrons.

\section{RESULTS}

\subsection{Snowballs in Euclid detectors}

After analyzing 600 hours of dark 2-hour data from Euclid Flight detectors, we found that the rate of occurrence of a snowball event is on average 1.34 snowballs per hour. If we remove snowballs that have central pixels that correlate with bad pixels in the operational mask (pixels that do not fulfill requirement for spectroscopy noise, photometry noise, linearity, and/or quantum efficiency) that value drops to 0.92 snowballs per hour.

Considering the size of the Euclid detectors (approximately 13.6 square centimeters), the rate of occurrence of a snowball event is 0.1 snowballs per hour per $\mathrm{cm}^{2}$ for all events, and 0.07 snowballs per hour per $\mathrm{cm}^{2}$ for events where the central pixels could not be associated with bad pixels in the operational mask.

As an example, Figure 1 shows the larger snowball that appeared in the detector SCS 18279_011-3_0020F038 during Flight Run 6. 


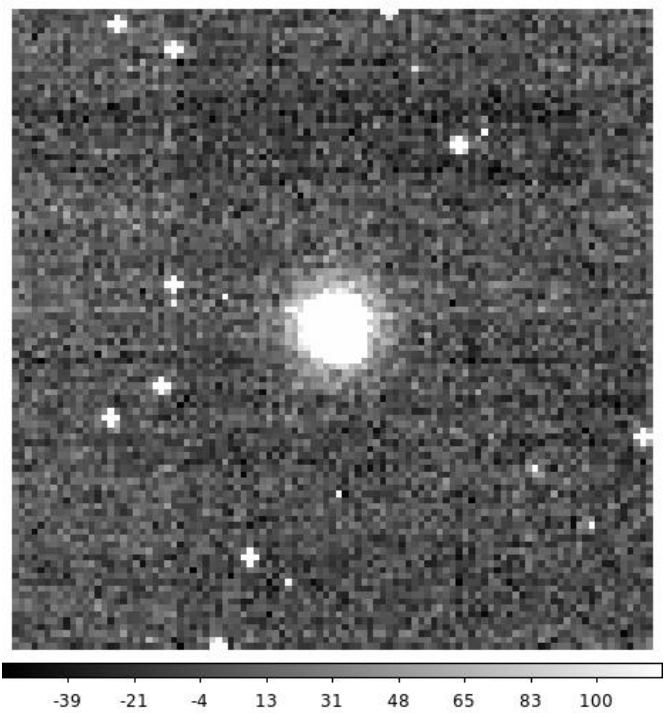

Figure 1. Snowball in Euclid detector SCS 18279_011-3_0020F038 (81 pixels affected, charged deposited $\sim 3 \times 10^{6}$ e-).

Table 1 outlines our results for each detector. We found that on some occasions a snowball event repeats in the same location. A snowball that repeats is more likely to occur in pixels that are associated with bad pixels in the operational mask, as seen by comparing the last two columns of Table 1. For the snowballs reported in Table 1, we could not find any visible features in the flat fields of the detectors at the snowball positions.

We could not find any correlation in the elapsed time between two snowballs that appear at the same position, and we noticed that on some occasions, there are repetitions but with less deposited charge than $10^{5}$ electrons.

Table 2 presents the average rate and standard deviation for each Flight Run, for snowballs that do not correlate with bad pixels in the operational mask. Notice that for Flight Run 2, we analyzed fewer detectors for fewer hours, so the discrepancy between this Flight Run and the others is larger, due to lack of enough statistics.

Figure 2 shows the position of snowball events for Euclid detectors in Flight Run 3, where we have more accumulated data. Blue dots correspond to the positions of snowballs that do not have central pixels associated with bad pixels in the operational mask, while orange dots correspond to snowballs with central pixels associated with bad pixels in the operational mask. The distribution of snowballs in these detectors is spatially homogeneous. 


\begin{tabular}{|c|c|c|c|c|c|c|c|c|}
\hline FR & detectors & $\begin{array}{l}\text { total } \\
\text { hours }\end{array}$ & \begin{tabular}{|c|}
$\begin{array}{c}\text { Number } \\
\text { of } \\
\text { snowballs }\end{array}$ \\
\end{tabular} & snowballs/hr & \begin{tabular}{|c|}
$\begin{array}{c}\text { Number of } \\
\text { snowballs without bad } \\
\text { pixels in op. mask }\end{array}$ \\
\end{tabular} & \begin{tabular}{|c|} 
snowballs/hr \\
without bad \\
pixels in op. mask
\end{tabular} & $\begin{array}{c}\text { Number of repeated } \\
\text { snowballs with bad } \\
\text { pixels in op. mask }\end{array}$ & $\begin{array}{c}\text { Number of repeated } \\
\text { snowballs without bad } \\
\text { pixels in op. mask }\end{array}$ \\
\hline FR1 & 18249_001-4_0008F015 & 8 & 20 & 2.50 & 9 & 1.13 & 8 & 4 \\
\hline FR1 & 18278_001-5_0010F014 & 8 & 8 & 1.00 & 3 & 0.38 & 0 & 0 \\
\hline FR1 & 18284_008-1_0012F016 & 8 & 29 & 3.63 & 7 & 0.88 & 23 & 0 \\
\hline FR1 & 18268_004-5_0014F017 & 8 & 5 & 0.63 & 4 & 0.50 & 0 & 0 \\
\hline FR2 & 18278_001-5_0013F043 & 8 & 10 & 1.25 & 7 & 0.88 & 3 & 0 \\
\hline FR2 & 18458_010-3_0015F019 & 8 & 37 & 4.63 & 19 & 2.38 & 17 & 2 \\
\hline FR3 & 18453_002-4_0019F037 & 60 & 113 & 1.88 & 77 & 1.28 & 29 & 18 \\
\hline FR3 & 18632_008-5_0020F038 & 60 & 52 & 0.87 & 52 & 0.87 & 0 & 0 \\
\hline FR3 & 18452_005-4_0021F042 & 60 & 43 & 0.72 & 41 & 0.68 & 2 & 0 \\
\hline FR3 & 18269_007-2_0023F048 & 60 & 52 & 0.87 & 51 & 0.85 & 0 & 0 \\
\hline FR4 & 18280_011-4_0019F037 & 40 & 45 & 1.13 & 45 & 1.13 & 0 & 1 \\
\hline FR4 & 18282_008-3_0020F038 & 40 & 56 & 1.40 & 50 & 1.25 & 3 & 10 \\
\hline FR4 & 18548_011-2_0021F042 & 40 & 45 & 1.13 & 38 & 0.95 & 6 & 9 \\
\hline FR4 & 18285_006-4_0023F048 & 40 & 25 & 0.63 & 25 & 0.63 & 0 & 0 \\
\hline FR5 & 18221_003-5_0019F037 & 20 & 16 & 0.80 & 16 & 0.80 & 0 & 0 \\
\hline FR5 & 18272_004-2_0020F038 & 20 & 15 & 0.75 & 15 & 0.75 & 0 & 0 \\
\hline FR5 & 18267_012-4_0021F042 & 20 & 12 & 0.60 & 11 & 0.55 & 0 & 0 \\
\hline FR5 & 18223_012-5_0023F048 & 20 & 22 & 1.10 & 22 & 1.10 & 0 & 2 \\
\hline FR6 & 18628_005-2_0019F037 & 24 & 16 & 0.67 & 13 & 0.54 & 2 & 0 \\
\hline FR6 & 18279_011-3_0020F038 & 24 & 21 & 0.88 & 21 & 0.88 & 0 & 2 \\
\hline FR6 & 18250_012-4_0023F048 & 24 & 26 & 1.08 & 23 & 0.96 & 3 & 6 \\
\hline & Total & 600 & 668 & 1.34 & 549 & 0.92 & 96 & 54 \\
\hline
\end{tabular}

Table 1. Snowballs in Euclid Detectors. Columns are: Total hours analyzed, total number of snowballs, snowballs per hour, total number of snowballs without central pixels associated with bad pixels in the operational mask, rate of snowballs without central pixels associated with bad pixels per hour, number of repeated snowballs with central pixels that correlate with bad pixels in the operational mask, number of repeated snowballs without central pixels that correlate with bad pixels in the operational mask.

\begin{tabular}{|c|c|c|}
\hline FR & snowballs/hour & standard dev. \\
\hline FR1 & 0.72 & 0.34 \\
FR2 & 1.63 & 1.06 \\
FR3 & 0.92 & 0.26 \\
FR4 & 0.99 & 0.27 \\
FR5 & 0.80 & 0.23 \\
FR6 & 0.79 & 0.22 \\
\hline
\end{tabular}

Table 2. Average rate of snowballs per hour and standard deviation for each Euclid Flight Run for snowballs that do not correlate with bad pixels in the operational mask. 
18453_002-4_0019F037

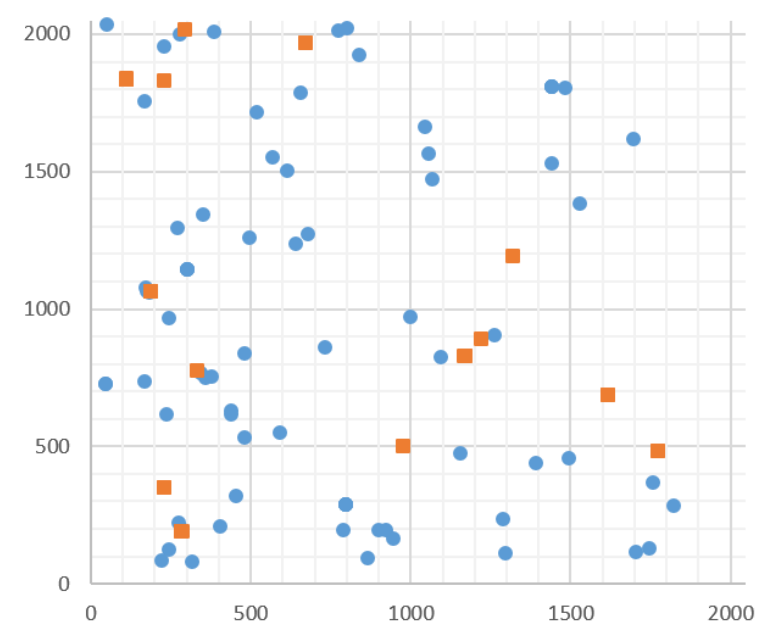

18452_005-4_0021F042

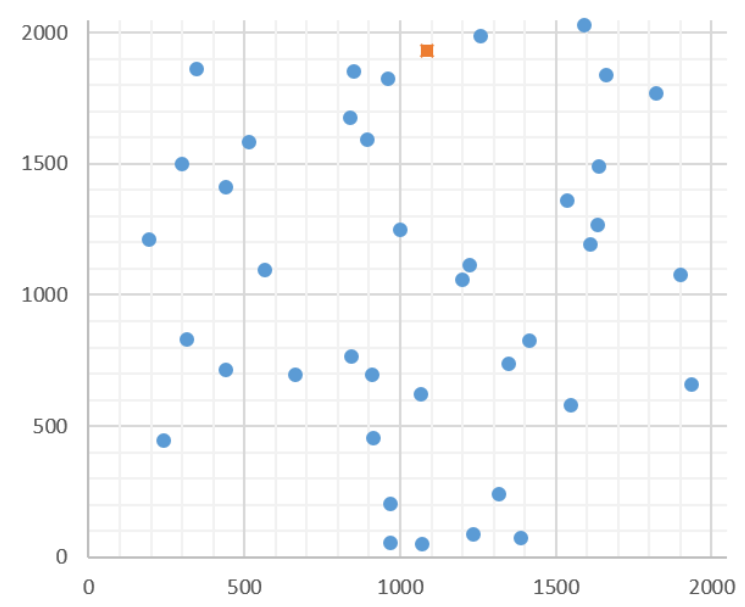

18632_008-5_0020F038

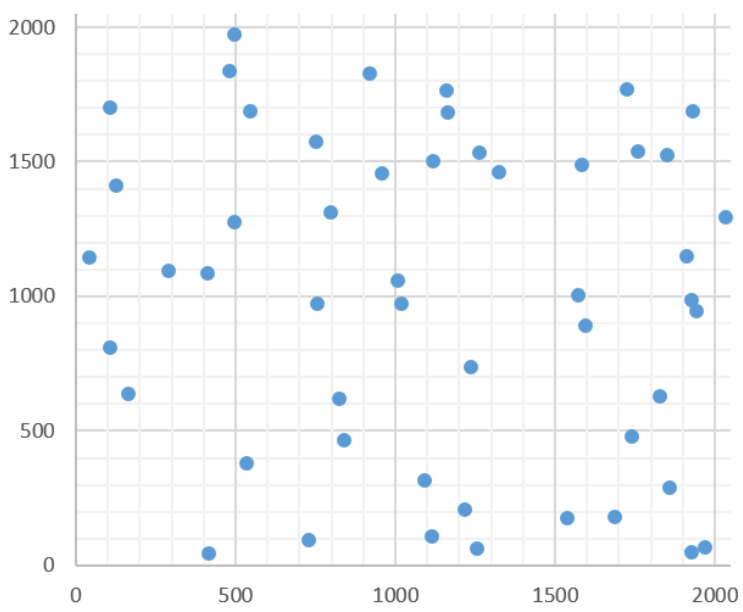

18269_007-2_0023F048

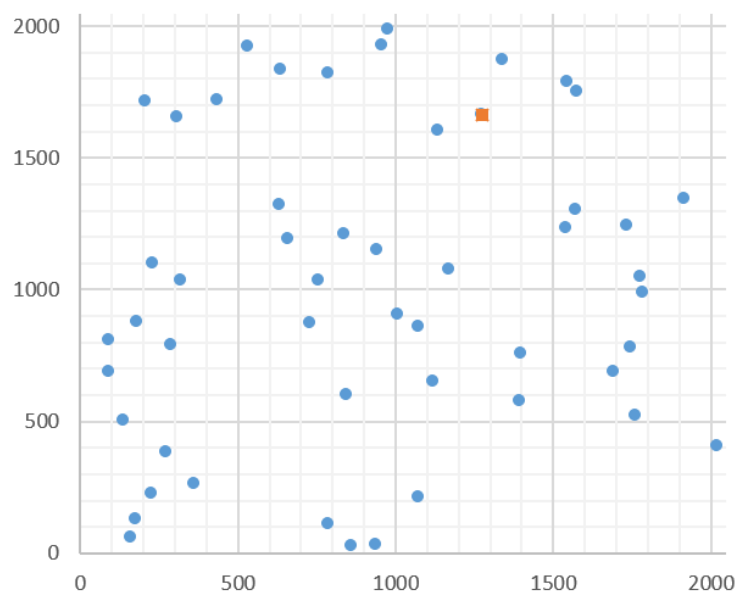

Figure 2. Spatial distribution of snowballs in detectors from Flight Run 3. Accumulation time: 60 hours.

The number of pixels affected in a snowball event (for charge deposited larger than $10^{5}$ electrons) was on average 25 pixels. The maximum number of pixels affected, 126 pixels, occurred in detector SCS18632_008-5_0020F038 during Flight Run 3. The total charge deposited in a snowball event had a median of $\sim 6 \times 10^{5}$ electrons with an average maximum between all Euclid detectors of about $3 \times 10^{6}$ electrons. Table 3 summarizes the results for each detector, with the total number of hours analyzed, mean, median, standard deviation, maximum of pixels affected, and charge deposited. Note that the numbers for the charge deposited in a snowball event reported here are lower limits. The central pixels in the snowballs could be saturated; they do not collect more charge after saturation. 


\begin{tabular}{|c|c|c|c|c|c|c|c|c|c|c|}
\hline \multirow{2}{*}{ FR } & \multirow{2}{*}{ detectors } & \multirow{2}{*}{$\begin{array}{l}\text { total } \\
\text { hours }\end{array}$} & \multicolumn{4}{|c|}{ pixels affected } & \multicolumn{4}{|c|}{ charged deposited [e-] } \\
\hline & & & mean & median & stddev & $\max$ & mean & median & stddev & $\max$ \\
\hline FR1 & 18249_001-4_0008F015 & 8 & 30.4 & 26 & 9.7 & 49 & $8.60 \mathrm{E}+05$ & $7.43 \mathrm{E}+05$ & $2.88 \mathrm{E}+05$ & $1.57 \mathrm{E}+06$ \\
\hline FR1 & 18278_001-5_0010F014 & 8 & 24.5 & 25 & 5.6 & 32 & $6.86 \mathrm{E}+05$ & $6.78 \mathrm{E}+05$ & $2.33 E+05$ & $1.11 \mathrm{E}+06$ \\
\hline FR1 & 18284_008-1_0012F016 & 8 & 27.3 & 29 & 7.7 & 44 & $7.77 \mathrm{E}+05$ & $7.31 E+05$ & $2.55 E+05$ & $1.15 \mathrm{E}+06$ \\
\hline FR1 & 18268_004-5_0014F017 & 8 & 21 & 20 & 7.2 & 28 & $6.09 E+05$ & $5.96 \mathrm{E}+05$ & $2.68 \mathrm{E}+05$ & $8.77 \mathrm{E}+05$ \\
\hline FR2 & 18278_001-5_0013F043 & 8 & 31.6 & 28 & 9.9 & 45 & $6.57 \mathrm{E}+05$ & $6.24 \mathrm{E}+05$ & $2.36 \mathrm{E}+05$ & $1.09 \mathrm{E}+06$ \\
\hline FR2 & 18458_010-3_0015F019 & 8 & 25.4 & 26 & 10.8 & 46 & $7.60 \mathrm{E}+05$ & $7.04 \mathrm{E}+05$ & $3.82 \mathrm{E}+05$ & $1.39 \mathrm{E}+06$ \\
\hline FR3 & 18453_002-4_0019F037 & 60 & 44.6 & 36.5 & 21.2 & 99 & $7.87 \mathrm{E}+05$ & $6.86 \mathrm{E}+05$ & $3.14 \mathrm{E}+05$ & $1.70 \mathrm{E}+06$ \\
\hline FR3 & 18632_008-5_0020F038 & 60 & 38.7 & 33 & 20.2 & 126 & $7.15 \mathrm{E}+05$ & $6.44 \mathrm{E}+05$ & $2.70 \mathrm{E}+05$ & $1.65 \mathrm{E}+06$ \\
\hline FR3 & 18452_005-4_0021F042 & 60 & 41.5 & 35 & 21.7 & 123 & $7.28 \mathrm{E}+05$ & $6.34 \mathrm{E}+05$ & $3.44 \mathrm{E}+05$ & $1.87 \mathrm{E}+06$ \\
\hline FR3 & 18269_007-2_0023F048 & 60 & 33.7 & 30.5 & 14.4 & 77 & $6.23 \mathrm{E}+05$ & $5.79 E+05$ & $2.36 \mathrm{E}+05$ & $1.20 \mathrm{E}+06$ \\
\hline FR4 & 18280_011-4_0019F037 & 40 & 24.1 & 22 & 8.5 & 48 & $7.00 E+05$ & $6.38 \mathrm{E}+05$ & $3.50 \mathrm{E}+05$ & $1.72 \mathrm{E}+06$ \\
\hline FR4 & 18282_008-3_0020F038 & 40 & 24.7 & 22 & 9.2 & 53 & $7.75 E+05$ & $6.80 \mathrm{E}+05$ & $3.24 \mathrm{E}+05$ & $1.79 E+06$ \\
\hline FR4 & 18548_011-2_0021F042 & 40 & 22.73 & 20 & 6.9 & 38 & $7.05 E+05$ & $5.90 \mathrm{E}+05$ & $2.76 \mathrm{E}+05$ & $1.27 \mathrm{E}+06$ \\
\hline FR4 & 18285_006-4_0023F048 & 40 & 21.48 & 21 & 4.7 & 34 & $6.67 \mathrm{E}+05$ & $6.19 E+05$ & $2.16 \mathrm{E}+05$ & $1.17 \mathrm{E}+06$ \\
\hline FR5 & 18221_003-5_0019F037 & 20 & 24.4 & 21 & 10.2 & 55 & $7.17 \mathrm{E}+05$ & $6.41 \mathrm{E}+05$ & $3.07 E+05$ & $1.50 \mathrm{E}+06$ \\
\hline FR5 & 18272_004-2_0020F038 & 20 & 20.3 & 19 & 6 & 37 & $5.90 \mathrm{E}+05$ & $4.92 \mathrm{E}+05$ & $2.45 \mathrm{E}+05$ & $1.21 \mathrm{E}+06$ \\
\hline FR5 & 18267_012-4_0021F042 & 20 & 20.7 & 21 & 6.3 & 30 & $6.45 \mathrm{E}+05$ & $6.30 E+05$ & $2.21 \mathrm{E}+05$ & $9.73 E+05$ \\
\hline FR5 & 18223_012-5_0023F048 & 20 & 24.8 & 21.5 & 13.3 & 70 & $7.25 \mathrm{E}+05$ & $6.71 \mathrm{E}+05$ & $4.13 \mathrm{E}+05$ & $2.19 \mathrm{E}+06$ \\
\hline FR6 & 18628_005-2_0019F037 & 24 & 22.2 & 19.5 & 8.4 & 45 & $6.60 \mathrm{E}+05$ & $5.70 \mathrm{E}+05$ & $2.57 \mathrm{E}+05$ & $1.25 \mathrm{E}+06$ \\
\hline FR6 & 18279_011-3_0020F038 & 24 & 24.7 & 20 & 14.3 & 81 & $8.08 \mathrm{E}+05$ & $6.23 E+05$ & $6.14 \mathrm{E}+05$ & $3.14 \mathrm{E}+06$ \\
\hline FR6 & 18250_012-4_0023F048 & 24 & 23 & 20 & 9.9 & 51 & $6.98 \mathrm{E}+05$ & $5.68 \mathrm{E}+05$ & $3.65 \mathrm{E}+05$ & $1.71 \mathrm{E}+06$ \\
\hline & Total: & 600 & 27.23 & 24.57 & 10.77 & 57.67 & $7.09 E+05$ & $6.35 \mathrm{E}+05$ & $3.05 \mathrm{E}+05$ & $1.50 \mathrm{E}+06$ \\
\hline
\end{tabular}

Table 3. Mean, Median, Standard Deviation, Maximum Number of pixels affected, and charge collected in a snowball event for each Euclid detector. Pixel pitch: $18 \mu \mathrm{m}$.

To compare between snowball events and cosmic ray hits, we looked for cosmic ray events during Flight Run 3 using the same data set used in our snowball search. For a charge deposited larger than $10^{5}$ electrons, Table 4 shows the number of snowballs and the number of cosmic rays found in each detector during 60 hours of data, while Table 5 compares the pixels affected. For the range of deposited charge we analyzed, cosmic rays hit the detector only $30 \%$ as often as snowballs events. In Figure 3 we plotted the deposited charge versus the number of pixels for each detector for snowball events and cosmic rays. From the mentioned figure (with the exception of a few cosmic ray events), the snowballs and the cosmic ray traces look like they come from different populations: snowballs need fewer pixels to collect the same amount of charge. As an example of a cosmic ray event, Figure 4 shows a huge cosmic ray that hit detector SCS 18269_007-2_0023F048 during Flight Run 3, with more than 300 pixels affected.

\begin{tabular}{|c|c|c|c|}
\hline $\begin{array}{c}\text { FR3 } \\
\text { detectors }\end{array}$ & $\begin{array}{c}\text { Total Number of } \\
\text { snowballs }\end{array}$ & $\begin{array}{c}\text { Number of snowballs } \\
\text { without bad px in the mask }\end{array}$ & $\begin{array}{c}\text { Total Number of } \\
\text { cosmic rays }\end{array}$ \\
\hline 18453_002-4_0019F037 & 113 & 77 & 11 \\
18632_008-5_0020F038 & 52 & 52 & 12 \\
18452_005-4_0021F042 & 43 & 41 & 20 \\
18269_007-2_0023F048 & 52 & 51 & 24 \\
\hline
\end{tabular}

Table 4. Total number of snowballs and cosmic ray events (with deposited charge larger than $10^{5}$ electrons) found during Flight Run 3. 
Deposited charge vs number of pixels affected Euclid detector 18453_002-4_0019F037

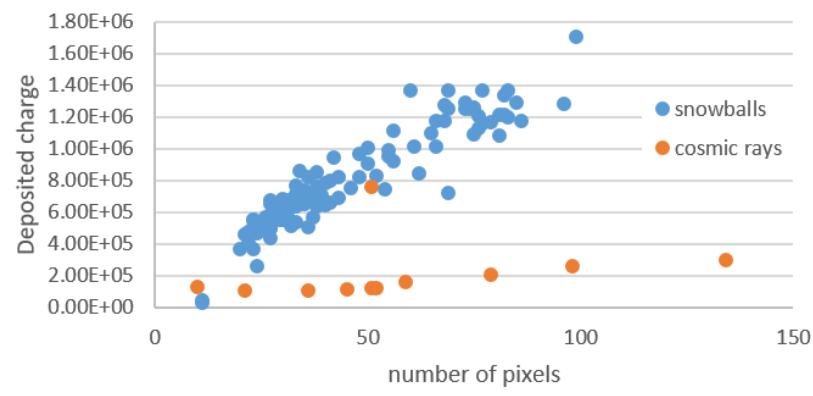

Deposited charge vs number of pixels affected Euclid Detector 18452_005-4_0021F042

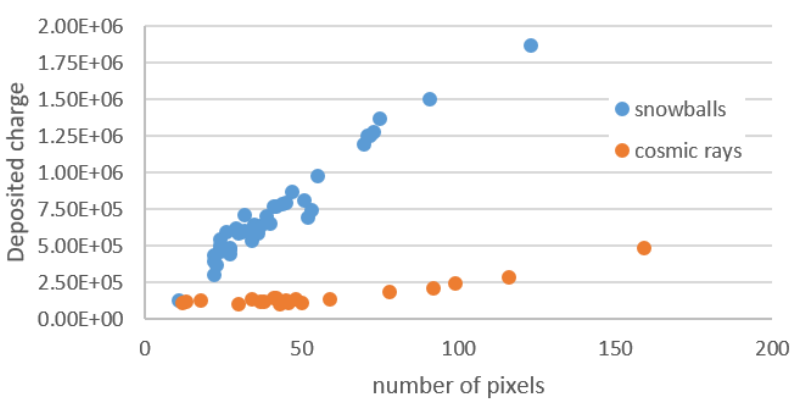

Deposited charge vs number of pixels affected Euclid detector 18632_008-5_0020F038

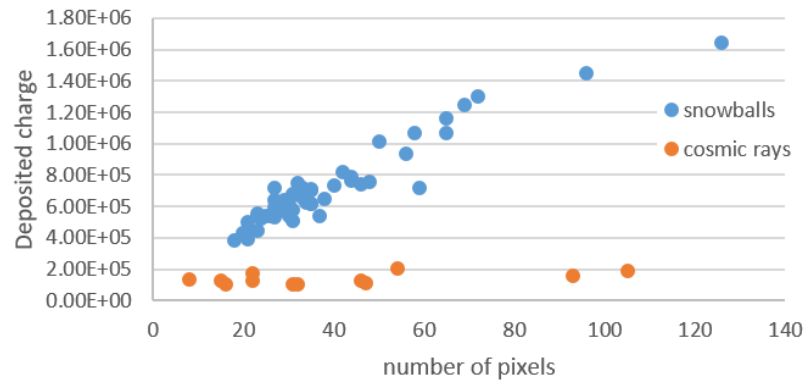

Deposited charge vs number of pixels affected Euclid detector 18269_007-2_0023F048

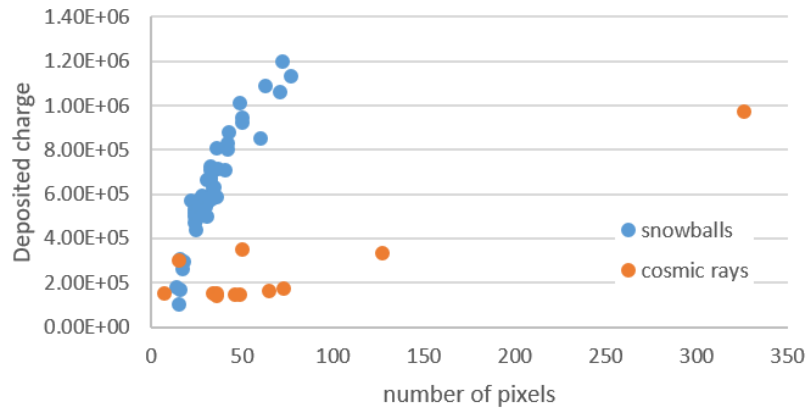

Figure 3. Deposited charge versus number of pixels affected for snowballs and cosmic ray events detected during Flight Run 3.

\begin{tabular}{|c|c|c|c|c|c|c|c|}
\hline $18453 \_002-4 \_00$ & $19 \mathrm{~F} 037$ & snowballs & cosmic rays & \multicolumn{2}{|c|}{ 18632_008-5_0020F038 } & snowballs & cosmic rays \\
\hline \multirow{4}{*}{ pixels affected } & mean: & 44.6 & 57.8 & \multirow{4}{*}{ pixels affected } & mean: & 38.7 & 40.9 \\
\hline & median: & 36.5 & 51.0 & & median: & 33.0 & 31.5 \\
\hline & sttdev: & 21.2 & 35.0 & & sttdev: & 20.2 & 30.6 \\
\hline & maximum: & 99.0 & 134.0 & & maximum: & 126.0 & 105.0 \\
\hline \multirow{4}{*}{ deposited charge [e-] } & mean: & $7.87 \mathrm{E}+05$ & $2.15 \mathrm{E}+05$ & \multirow{4}{*}{ deposited charge [e-] } & mean: & $7.15 E+05$ & $1.38 \mathrm{E}+05$ \\
\hline & median: & $6.86 \mathrm{E}+05$ & $1.28 \mathrm{E}+05$ & & median: & $6.44 \mathrm{E}+05$ & $1.26 \mathrm{E}+05$ \\
\hline & sttdev: & $3.14 \mathrm{E}+05$ & $1.92 \mathrm{E}+05$ & & sttdev: & $2.70 E+05$ & $3.48 \mathrm{E}+04$ \\
\hline & maximum: & $1.70 \mathrm{E}+06$ & $7.59 \mathrm{E}+05$ & & maximum: & $1.65 \mathrm{E}+06$ & $2.02 \mathrm{E}+05$ \\
\hline \multicolumn{2}{|c|}{ 18452_005-4_0021F042 } & snowballs & cosmic rays & \multicolumn{2}{|c|}{ 18269_007-2_0023F048 } & snowballs & cosmic rays \\
\hline \multirow{4}{*}{ pixels affected } & mean: & 41.5 & 55.0 & \multirow{4}{*}{ pixels affected } & mean: & 33.7 & 51.7 \\
\hline & median: & 35.0 & 44.0 & & median: & 30.5 & 36.0 \\
\hline & sttdev: & 21.7 & 36.9 & & sttdev: & 14.4 & 63.3 \\
\hline & maximum: & 123.0 & 159.0 & & maximum: & 77.0 & 326.0 \\
\hline \multirow{4}{*}{ deposited charge [e-] } & mean: & $7.28 \mathrm{E}+05$ & $1.64 \mathrm{E}+05$ & \multirow{4}{*}{ deposited charge [e-] } & mean: & $6.23 E+05$ & $1.92 \mathrm{E}+05$ \\
\hline & median: & $6.34 \mathrm{E}+05$ & $1.32 \mathrm{E}+05$ & & median: & $5.79 \mathrm{E}+05$ & 1. $43 \mathrm{E}+05$ \\
\hline & sttdev: & $3.44 \mathrm{E}+05$ & $8.92 \mathrm{E}+04$ & & sttdev: & $2.36 \mathrm{E}+05$ & $1.79 \mathrm{E}+05$ \\
\hline & maximum: & $1.87 \mathrm{E}+06$ & $4.83 \mathrm{E}+05$ & & maximum: & $1.20 \mathrm{E}+06$ & $9.70 \mathrm{E}+05$ \\
\hline
\end{tabular}

Table 5. Comparison between snowballs and cosmic ray events during Flight Run 3 for snowballs and cosmic rays with deposited charge larger than $10^{5}$ electrons: Number of pixels affected and deposited charge.

As other authors reported before for $\mathrm{HgCdTe}$ detectors $^{1}$, the reference pixels were not affected by a snowball event or a cosmic ray hit. We show examples of that in Figure 5. 


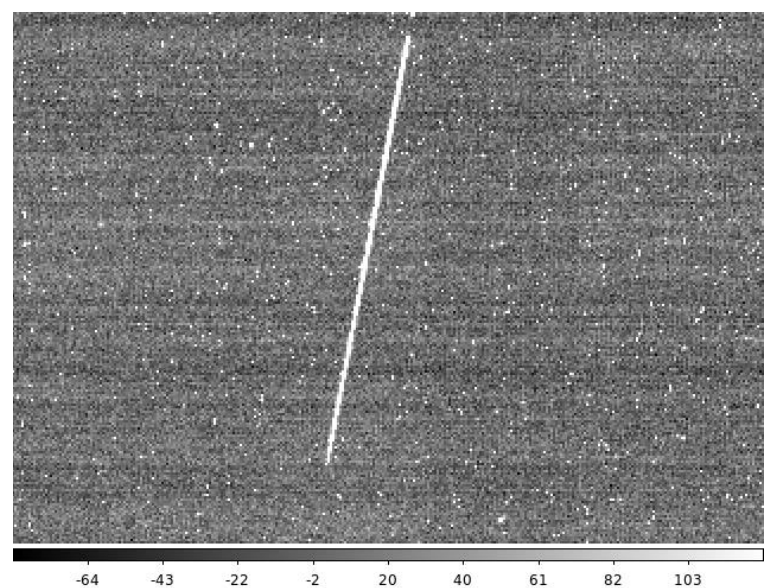

Figure 4. A cosmic ray hitting detector SCS 18269_007-2_0023F048 during Flight Run 3 with more than 300 pixels affected.

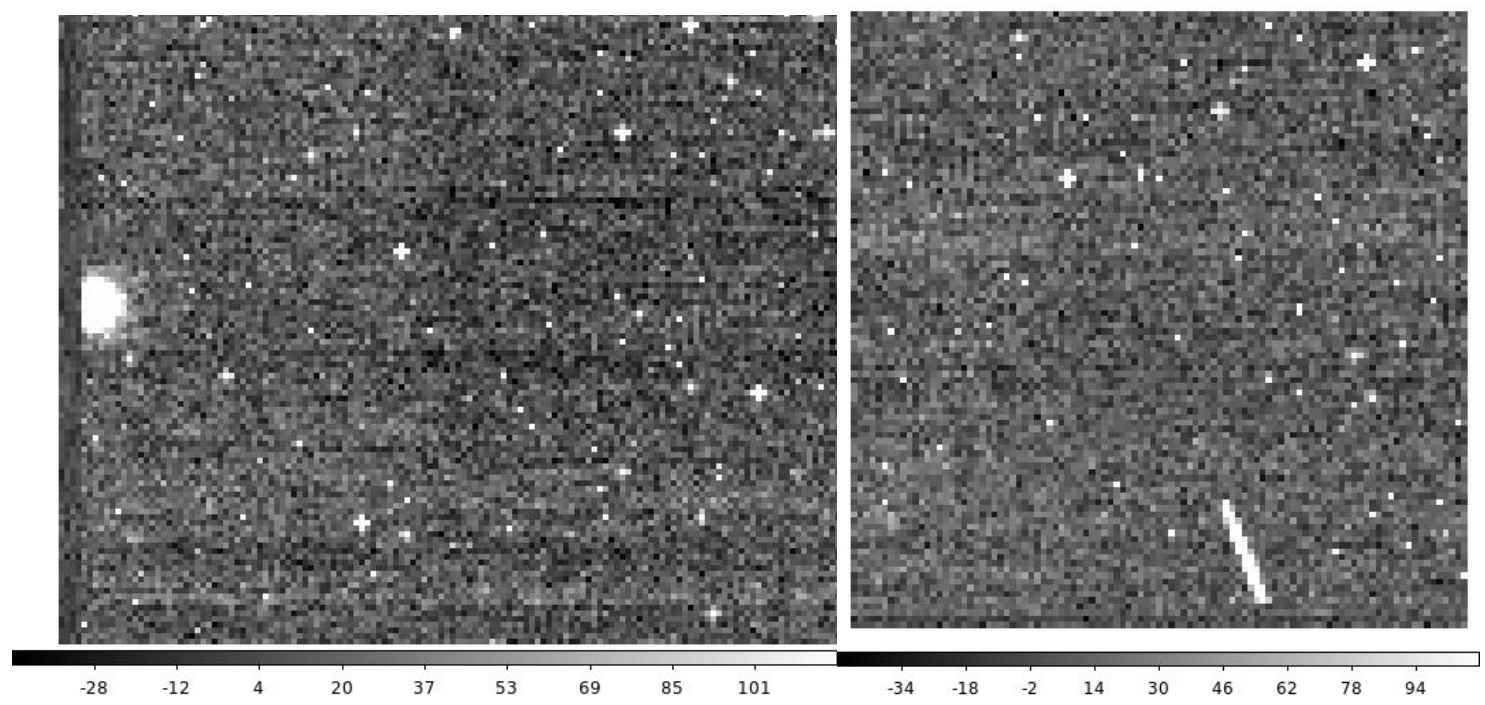

Figure 5. Example of snowball (left) and cosmic ray (right) not affecting reference pixels. The images correspond to detector SCS 18282_008-3_0020F038 during Flight Run 4 (left) and SCS 18453_002-4_0019F037 during Flight Run 3.

It is worth mentioning that in our Euclid Flight Run characterization at the DCL, we removed cosmic rays in our spectroscopy and photometry noise analysis. To that end, we computed the pixel slopes for each one of the 100 exposures taken to perform the noise analysis; we calculated the median and the standard deviation among the 100 exposures for each pixel. After that, for each pixel, we computed the number of the exposures where the value of the pixel is six times larger than the standard deviation. If that number was between one and three, the value of the pixel (in the exposures where the number is larger than 6 times the standard deviation) was replaced by its median value over the 100 exposures. If that number was higher than three, then no correction was applied. As you can see in Figure 6 snowballs are not totally removed from the spectroscopy noise result where the mentioned algorithm was applied. 


\section{Before correction}

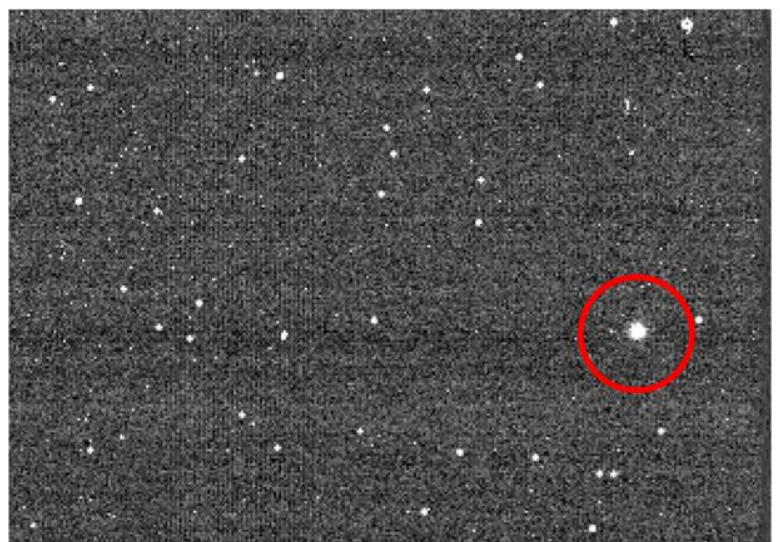

\section{After correction}

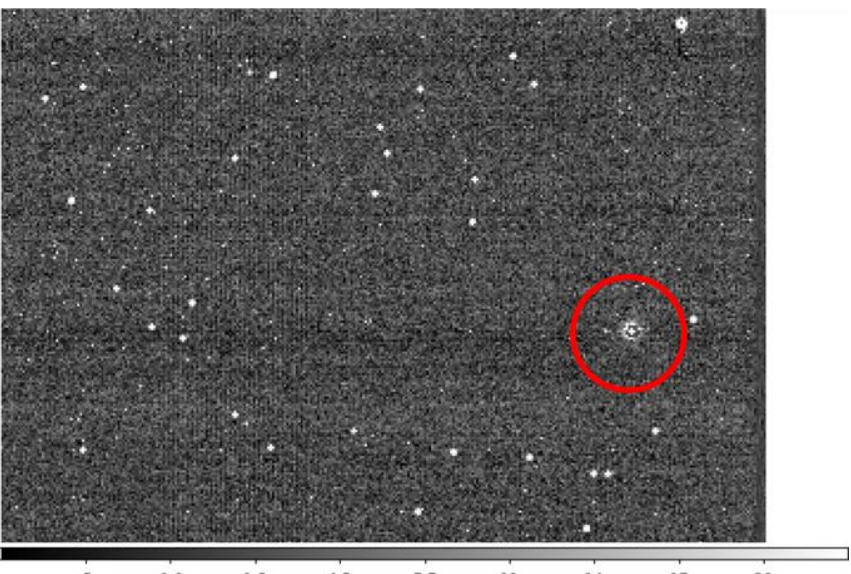

Figure 6. Example of a snowball that appears in spectroscopy noise analysis in Euclid Flight Run 1 in the detector SCS 18278_001-5_0010F014. Before (left) and after (right) applying the algorithm of "noise" removal.

\subsection{Results for WFIRST detectors}

For WFIRST detectors, we performed our analysis using multiple engineering detectors from the PV3 Full Array Lot and one PV2A detector (18138) that was tested several times at the DCL.

In Table 6 we report our results for the snowballs found in PV3 Full Array Lot detectors. Analyzing a total of 254 hours of detector data, we found 186 snowballs. This corresponds to an average between all PV3 Full Array Lot detectors of 0.70 snowballs per hour, or 0.04 snowballs per hour per $\mathrm{cm}^{2}$, but we saw variation between detectors. As in Euclid detectors, we found snowballs that repeat at the same spatial localization. Without taking into account the snowballs that repeat, the rate of occurrence of a snowball event in these detectors dropped to 0.43 snowballs per hour, or 0.03 snowballs per hour per $\mathrm{cm}^{2}$.

Figure 7 shows an example of a snowball found in detector 18237, where 83 pixels were affected and the total deposited charge was $5.7 \times 10^{5}$ electrons. 


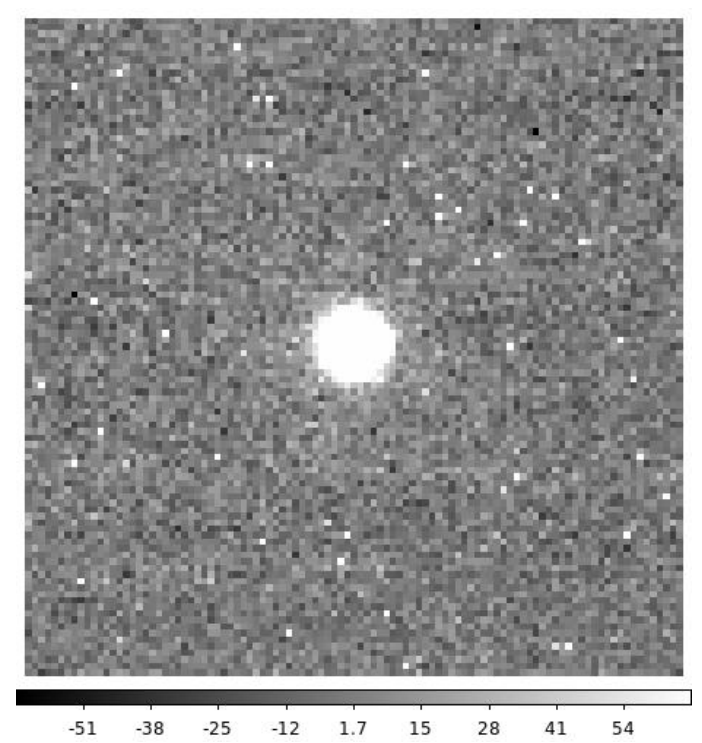

Figure 7. Snowball in WFIRST PV3 detector 18237 (83 pixels, charge deposited 5.7e5 e-).

\begin{tabular}{|c|c|c|c|c|c|}
\hline Detector & $\begin{array}{l}\text { total } \\
\text { hours }\end{array}$ & $\begin{array}{r}\text { Number of } \\
\text { snowballs }\end{array}$ & \begin{tabular}{|c|} 
total \\
snowballs/hr
\end{tabular} & \begin{tabular}{|c|}
$\begin{array}{c}\text { Number of } \\
\text { non-repeated } \\
\text { snowballs }\end{array}$ \\
\end{tabular} & $\begin{array}{c}\text { non-repeated } \\
\text { snowballs/hr }\end{array}$ \\
\hline 18237 & 26 & 2 & 0.08 & 2 & 0.08 \\
\hline 18238 & 14 & 7 & 0.50 & 7 & 0.50 \\
\hline 18239 & 8 & 3 & 0.38 & 1 & 0.13 \\
\hline 18240 & 14 & 3 & 0.21 & 3 & 0.21 \\
\hline 18241 & 28 & 24 & 0.86 & 22 & 0.79 \\
\hline 18242 & 8 & 3 & 0.38 & 3 & 0.38 \\
\hline 18243 & 14 & 32 & 2.29 & 17 & 1.21 \\
\hline 18244 & 22 & 9 & 0.41 & 7 & 0.32 \\
\hline 18438 & 40 & 27 & 0.68 & 11 & 0.28 \\
\hline 18440 & 40 & 54 & 1.35 & 17 & 0.43 \\
\hline 18443 & 40 & 22 & 0.55 & 15 & 0.38 \\
\hline Total & 254 & 186 & 0.70 & 105 & 0.43 \\
\hline
\end{tabular}

Table 6. Snowballs in PV3 Full Array Lot detectors: Total hours analyzed, total number of snowballs, snowballs per hour, number of non-repeated snowballs, non-repeated snowballs per hour.

As for Euclid detectors, we recorded the number of pixels affected and the charge deposited. Table 7 shows the results for each detector, with an average of about 42 pixels and a maximum of 112 pixels affected in detector 18438 . 


\begin{tabular}{|c|c|c|c|c|c|c|c|c|c|}
\hline \multirow{2}{*}{ detectors } & \multirow{2}{*}{$\begin{array}{l}\text { total } \\
\text { hours }\end{array}$} & \multicolumn{4}{|c|}{ pixels affected } & \multicolumn{4}{|c|}{ charged deposited [e-] } \\
\hline & & mean & median & stddev & $\max$ & mean & median & stddev & $\max$ \\
\hline 18237 & 26 & 59.5 & 59.5 & 33.2 & 83 & $4.08 \mathrm{E}+05$ & $4.08 \mathrm{E}+05$ & $2.34 \mathrm{E}+04$ & $5.74 \mathrm{E}+05$ \\
\hline 18238 & 14 & 48.3 & 33.0 & 28.6 & 100 & $2.86 \mathrm{E}+05$ & $2.09 E+05$ & $1.27 \mathrm{E}+05$ & $4.92 \mathrm{E}+05$ \\
\hline 18239 & 8 & 34.7 & 35.0 & 8.5 & 43 & $1.76 \mathrm{E}+05$ & $1.97 \mathrm{E}+05$ & $5.84 \mathrm{E}+04$ & $2.21 \mathrm{E}+05$ \\
\hline 18240 & 14 & 34.0 & 32.0 & 14.1 & 49 & $2.34 \mathrm{E}+05$ & $2.49 E+05$ & $1.01 \mathrm{E}+05$ & $3.28 \mathrm{E}+05$ \\
\hline 18241 & 28 & 37.5 & 33.0 & 15.4 & 85 & $2.00 \mathrm{E}+05$ & $1.83 \mathrm{E}+05$ & $6.24 \mathrm{E}+04$ & $3.96 \mathrm{E}+05$ \\
\hline 18242 & 8 & 38.0 & 39.0 & 16.5 & 54 & $2.45 \mathrm{E}+05$ & $2.37 E+05$ & $1.04 \mathrm{E}+05$ & $3.54 \mathrm{E}+05$ \\
\hline 18243 & 14 & 37.2 & 33.0 & 15.1 & 88 & $2.20 \mathrm{E}+05$ & $2.05 E+05$ & $8.51 \mathrm{E}+04$ & $4.82 \mathrm{E}+05$ \\
\hline 18244 & 22 & 61.0 & 60.0 & 24.1 & 102 & $3.50 \mathrm{E}+05$ & $3.58 \mathrm{E}+05$ & $1.27 \mathrm{E}+05$ & $5.55 \mathrm{E}+05$ \\
\hline 18438 & 40 & 39.6 & 32.0 & 21.4 & 112 & $2.15 \mathrm{E}+05$ & $2.14 \mathrm{E}+05$ & $6.50 \mathrm{E}+04$ & $3.16 \mathrm{E}+05$ \\
\hline 18440 & 40 & 36.1 & 32.5 & 13.1 & 66 & $1.89 \mathrm{E}+05$ & $1.69 \mathrm{E}+05$ & $6.68 \mathrm{E}+04$ & $3.27 E+05$ \\
\hline 18443 & 40 & 34.8 & 35.0 & 8.5 & 51 & $2.01 \mathrm{E}+05$ & $1.95 \mathrm{E}+05$ & $6.44 \mathrm{E}+04$ & $3.01 \mathrm{E}+05$ \\
\hline Total & 254 & 41.9 & 38.5 & 18.0 & 76 & $2.48 \mathrm{E}+05$ & $2.39 \mathrm{E}+05$ & $8.04 \mathrm{E}+04$ & $3.95 E+05$ \\
\hline
\end{tabular}

Table 7. Mean, Median, Standard Deviation, Maximum Number of pixels affected, and charge collected in a snowball event for each PV3 Full Array Lot detector. Pixel pitch: $10 \mu \mathrm{m}$.

In Figure 8 we show the spatial distribution of the snowballs found in detectors 18438 and 18443. Both detectors were tested together in the HyC Dewar at the DCL, and the pictures correspond to a total time of 40 hours.
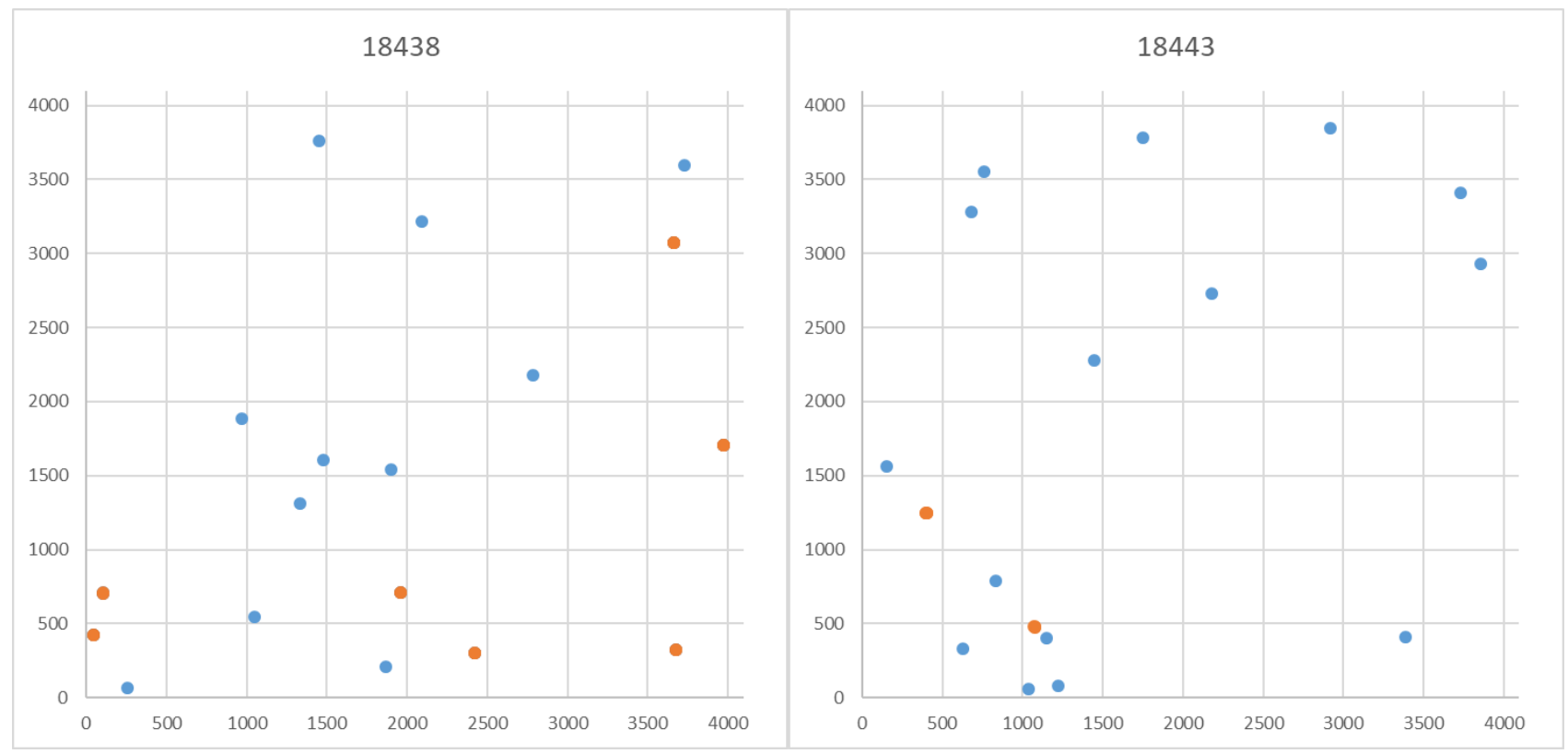

Figure 8. Spatial distribution of snowballs in detectors 18438 and 18443 (WFIRST). The blue dots correspond to the snowballs that do not repeat. The orange dots correspond to the snowballs that repeat. Accumulation time: 40 hours.

We evaluated if the repeated snowballs were associated with features in the flat fields of the detectors. In detectors 18239 and 18241, one snowball repeated, and we could not find anything evident in the flat field. In detector 18243 there is a small feature in the flat field in the snowball that repeated several times (in different exposures). In detector 18244, close to the snowball that repeated there is a large feature. In detector 18438, several snowballs repeated, and in one of then there is a large feature in the flat field that reaches the snowball region. In detector 18440, there were several 
snowballs that repeat, but there were no evident features in the flat fields. Finally in detector 18443 , there are 2 snowballs that repeated with nothing evident in the flat field.

For the PV2A detector (18138) we searched for snowballs in the six occasions this detector was tested between August 2016 and May 2017 in the HyC Dewar at the DCL. The snowball rate in this detector was low (0.28 snowballs per hour) and we could not see a decrease or increase in the snowball appearance during the time that was tested. Table 8 summarizes the results of the different runs where the detector was evaluated. Three snowballs repeated in this detector. Two snowballs repeated twice and one snowballs repeated three times. These repetitions occurred during the same run: two of them during the same exposure, and one of them in two consecutive exposures.

\begin{tabular}{|r|c|c|c|c|}
\hline date & $\begin{array}{c}\text { total } \\
\text { hours }\end{array}$ & $\begin{array}{c}\text { total } \\
\text { snowballs }\end{array}$ & $\begin{array}{c}\text { snowballs/hr } \\
\text { hours }\end{array}$ & repetitions \\
\hline Aug-2016 & 18 & 6 & 0.33 & 0 \\
Sep-2016 & 26 & 6 & 0.23 & 0 \\
Oct-2016 & 10 & 3 & 0.30 & 0 \\
Nov-2016 & 12 & 2 & 0.17 & 0 \\
Apr-2017 & 18 & 12 & 0.67 & $3(2,2,3)$ \\
May-2017 & 10 & 0 & 0.00 & 0 \\
\hline total & 94 & 29 & 0.28 & 3 \\
\hline
\end{tabular}

Table 8. Snowballs in PV2A detector 18138: Total hours analyzed, total number of snowballs, snowballs per hour, number of repeated snowballs (with number of repetitions for each case).

\section{DISCUSSION}

In this work, we searched for snowballs in Euclid and WFIRST detectors. After analyzing 600 hours of dark data from Euclid Flight Detectors, we found that the rate of occurrence of a snowball event (for a deposited charge larger than $10^{5}$ electrons) is on average 1.34 snowballs per hour. If we do not consider the snowballs that have central pixels associated with bad pixels in the operational mask, the value drops to 0.92 snowballs per hour. For the size of the Euclid detectors, these values correspond to a rate of 0.1 snowballs per hour per $\mathrm{cm}^{2}$ for all events, and 0.07 snowballs per hour per $\mathrm{cm}^{2}$ for events where the central pixels are not associated with bad pixels in the operational mask.

For WFIRST detectors we looked into 254 hours of dark data from the PV3 Full Array Lot and 94 hours of data from PV2A detector 18138. On average between all PV3 Full Arrays Lot detectors, we found a rate of 0.70 snowballs per hour, or 0.04 snowballs per hour per $\mathrm{cm}^{2}$, but we saw a significant variation between detectors. We found some snowballs that repeat at the same spatial location. Without taking into account the snowballs that repeat, the rate of occurrence of a snowball event in WFIRST detectors dropped to 0.43 snowballs per hour or 0.03 snowballs per hour per $\mathrm{cm}^{2}$.

NIRCam detectors are comparable in size with Euclid detectors, and WFC3 detectors are 1/4 of the previous detectors in size. That implies that we have found, on average per area, about twice as many snowballs in Euclid detectors compared to NIRCam ${ }^{4}$ detectors, and about one half as many as found in WFC $3^{1}$ detectors. For WFIRST detectors (about $20 \%$ larger in size than Euclid detectors), the snowball rate per area was very similar to that found in NIRCam detectors ${ }^{4}$.

For Euclid we collected 10 frames in each 2-hour dark data file, while for WFIRST we collected 101 frames. Interestedly, the rate in WFIRST detectors is lower than in Euclid detectors. The sampling time or number of frames collected for the same total exposure time does not appear to be correlated to an increase or decrease in the number of snowballs in a detector. 
The charge deposited by snowballs in Euclid and WFIRST detectors corresponds to the same range as the ones reported in WFC $3^{1}$ and NIRCam ${ }^{4}$ detectors. We observed that in particular, for WFIRST detectors, the spread in the deposited charge between detectors is very narrow. The deposited charge ranged on average between $\sim 1.8 \times 10^{5}$ and $\sim 4.1 \times 10^{5}$ electrons for all PV3 Full Array Lot detectors. This is in line with the hypothesis of alpha particles, products of a decay of radiative contaminants in the detector material, as sources of snowballs events ${ }^{2}$. The alpha particles are emitted with little range of variation about $4 \mathrm{MeV}$. We have to consider that the numbers reported here for the collected charge are lower limits; the central pixels of the snowballs are saturated.

The number of pixels affected in Euclid detectors was on average smaller than for WFIRST detectors. This may be related, at least in part, to the crosstalk difference between the detectors analyzed: the average of the nearest neighbors in the inter-pixel capacitance matrix for the Euclid detectors tested in this work was 0.657\%, while for the PV3 Full Array Lot WFIRST detectors, the average was $2.098 \%$. However, when we take into account the pixel pitch, a snowball in Euclid detectors is almost double the size of a snowball found in WFIRST detectors. For NIRCam, the area affected was even larger (250-400 pixels of $18 \mu \mathrm{m}$ pitch) $)^{4}$.

As in WFC $3^{1}$ and NIRCam ${ }^{4}$ we found snowballs that repeat at the same spatial location in both Euclid and WFIRST detectors. It is more likely to find a snowball that repeats if the central pixels are associated with a bad pixel in the operational mask in the case of Euclid detectors. For WFIRST detectors, on some occasions we were able to see features in the flat fields close to the snowballs that repeat.

McCullough ${ }^{2}$ discussed extensively the possible source of repeated snowballs as a daughter of the radiative decay of the U-238 and Th-232 chains. He also claimed that If Th-232 or Th-238 decays create snowballs then the snowball events will be grow with time by a factor 2 to 4 in 10 years. With the data analyzed in this work, we cannot establish if the number of the snowballs increases, decreases or is stable over time in Euclid or WFIRST detectors.

With the exception of WFIRST detector 18138, where we searched for snowballs in data taken at different times over the course of one year, the data used in this work was mostly taken for each detector during the same day. Consequently, assuming a hypothesis of radioactive elements as sources of snowballs, it is hard to conclude if the elapsed time between the snowballs that repeat are associated with a particular radioactive chain. In the case of 18138, the repetitions occurred only at the same exposures, or in different exposures but with a difference of a few hours. For other detectors as well, most of the repetitions occurred during the same exposure or in exposures taken during the same day.

It was also discussed previously ${ }^{1}$, that cosmic rays (muons in particular), which reach sea level at a rate of 1 particle per square centimeter per minute, could be the origin of the snowballs. We analyzed that option in the Euclid detectors of Flight Run 3. We found that when comparing the snowballs and cosmic rays in those detectors, the cosmic rays need more pixels in order to collect the same amount of charge, suggesting that these two events may be originated by different sources. Besides, for snowballs that repeat at the same localization, it would be almost impossible to assign them a cosmic ray origin.

We found snowballs that appeared close to the reference pixels in Euclid detectors, and we verified that those pixels were not affected. Unfortunately, in our snowball search in WFIRST detectors, no snowball appeared close to the reference pixels in any occasion.

Finally, we do not expect the snowballs to significantly affect science data in Euclid or WFIRST detectors. First, the rate of occurrence is low; second, it is expected that at least some of the snowballs will appear in pixels already rejected because they do not fulfill expectations; and third, at least part of the snowballs could be removed by noise rejecting algorithms.

The coordinates of the snowballs reported in this work could be available upon request. 


\section{REFERENCES}

[1] Bryan Hilbert, "Snowballs" in the WFC3-IR Channel: Characterization, Instrument Science Report WFC3 2009-43, (2009).

[2] Peter McCullough, Radioactivity in HgCdTe devices: potential source of "snowballs", Instrument Science Report WFC3 2009-44 (2009).

[3] M. J. Durbin, M. Bourque, S. Baggett, IR "Snowballs": Long-Term Characterization, Space Telescope Science Institute Instrument Science Report WFC3 2015-01 (2015).

[4] Bernard J. Rauscher, et al., Publications of the Astronomical Society of the Pacific, 126:739-749, (2014).

[5] Bryan Hilbert, Technical Report, Doc \#: JWST-STScI-004407, SM-12 (2015)

[6] David. A. Cottingham, Internal Report, Detector Characterization Laboratory, Goddard Space Flight Center, NASA (2017). 